\title{
Nineteen Years of Japanese Encephalitis Surveillance in Sabah, Malaysian Borneo
}

\author{
Marilyn Charlene Montini Maluda, ${ }^{1,2}$ Jenarun Jelip, ${ }^{3}$ Mohd Yusof Ibrahim, ${ }^{1}$ Maria Suleiman, ${ }^{3}$ Mohammad Saffree Jeffree, ${ }^{1,4}$ \\ Azalina Farina Binti Aziz, ${ }^{4}$ Jaeyres Jani, ${ }^{4}$ Takaaki Yahiro, ${ }^{5}$ and Kamruddin Ahmed ${ }^{4,5 *}$ \\ ${ }^{1}$ Department of Community and Family Medicine, Faculty of Medicine and Health Sciences, University Malaysia Sabah, Kota Kinabalu, Malaysia; \\ ${ }^{2}$ Sabah State Health Department, Kota Kinabalu, Malaysia; ${ }^{3}$ Ministry of Health Malaysia, Putrajaya, Malaysia; ${ }^{4}$ Borneo Medical and Health Research \\ Centre, Kota kinabalu, Malaysia; ${ }^{5}$ Department of Pathobiology and Medical Diagnostics, Faculty of Medicine and Health Sciences, Universiti \\ Malaysia Sabah, Kota Kinabalu, Malaysia
}

\begin{abstract}
Japanese encephalitis (JE) is endemic in Malaysia. Although JE vaccination is practiced in the neighboring state of Sarawak for a long time, little is known about JE in Sabah state in Borneo. As a result, informed policy formulation for JE in Sabah has not been accomplished. In the present study, we have analyzed JE cases that have been reported to the Sabah State Health Department from 2000 to 2018. A total of 92 JE cases were reported during 19 years, and threefourths of the cases were attributed to children. The estimated mean incidence for JE cases is $0.161 / 100,000$ population. Japanese encephalitis was predominant in Sabah during June, July, and August, peaking in July. In most cases, pigs were absent within a 400-m radius of the place of residence. We could not establish any relationship between the mapping of JE cases and the number of piggeries in each district. We could not establish a relationship between average rainfall and JE cases, either. We propose the cases reported are possibly showing the tip of an iceberg and continuous surveillance is needed, as JE is a public health challenge in Sabah.
\end{abstract}

\section{INTRODUCTION}

Globally, every year 68,000 cases of encephalitis occur in humans because of the Japanese encephalitis virus (JEV). ${ }^{1}$ Malaysia is among the 24 countries endemic to JEV transmission. ${ }^{1}$ All five genotypes of JEV have been detected in Malaysia. As a result, it has been postulated that JEV has originated in the Malaysian/Indonesian region. ${ }^{2}$ Malaysia is a federation of 13 states and three federal territories, 11 states and two federal territories are in Peninsular Malaysia, and two states (Sarawak and Sabah) and one federal territory are in Borneo. Japanese encephalitis has been well documented in Peninsular Malaysia and Sarawak; however, the distribution of human JE in Sabah is unknown. Whereas the presence of JEV antibodies has been determined in pigs, sheep, ${ }^{3}$ and even in orangutans ${ }^{4}$ in Sabah, the seroprevalence of JEV in humans is unknown as it is assumed that Sabah is not endemic for human JE. Possibly because of this, no studies have been conducted on JE in Sabah so far. Therefore, it is challenging to retrieve information on the topic. Although there are vaccines available for JE, currently they are not part of the routine immunization program in Sabah and they are not available in the private healthcare systems. This study aims to generate evidence that will contribute to policy discussions by the relevant authorities on JE control and vaccination. Therefore, the objective of this study was to explore the epidemiology of JE in Sabah based on population surveillance data collected over a 19-year period.

\section{METHODOLOGY}

Study setting and population. This is a retrospective observational study reviewing all JE cases occurred in Sabah from January 2000 through December 2018, notified to the Vector Borne Disease Control Division, Sabah State Health

\footnotetext{
*Address correspondence to Kamruddin Ahmed, Department of Pathobiology and Medical Diagnostics, Faculty of Medicine and Health Sciences, Universiti Malaysia Sabah, Kota Kinabalu 88400, Malaysia. E-mail: ahmed@ums.edu.my
}

Department. The study protocol was approved by the Medical Ethics and Research Committee, Ministry of Health, Malaysia. The study protocol was registered under the National Medical Research Registry (NMRR-18-2767-44385).

Definition of epidemiological and clinical variables. Case definition for JE was determined according to the WHO guidelines. ${ }^{5}$ All cases were confirmed by anti-JEV IgM detection, and/or by reverse transcription-PCR (RT-PCR) of the serum and/or cerebrospinal fluid. Anti-JEV IgM was detected by JE Detect ${ }^{\mathrm{TM}}$ IgM Antibody Capture ELISA (InBios International Inc., Seattle, WA) according to the instructions of the manufacturer. Reverse transcription-PCR was performed according to published methods. ${ }^{6}$ These tests were performed at the National Public Health Laboratory, Selangor, Malaysia. All cases were categorized into three age-groups, namely, children younger than 15 years, adults aged 15-59 years, and elderly older than 60 years.

Mapping. QGIS 2.16.2 software (www.qgis.org/en/site) was used to illustrate reported JE cases and pig farms in different districts of Sabah. Data on the piggeries were obtained from the Sabah State Veterinary Department.

Correlation between rainfall and JE cases. Average rainfall data for each district of Sabah were obtained from the meteorological office. Average rainfall in each district was plotted against the cumulative number of JE cases in respective districts to find out the correlation between average rainfall and the number of JE cases.

Statistical analysis. Data were compiled and analyzed using GraphPad. All data were anonymized.

\section{RESULTS}

From 2000 through 2018, a total of 92 cases of JE have been confirmed. Data on age and gender were available from 2005 onward; during this period, 56 confirmed JE cases were detected. There were 28 males and 28 females, with a mean age of 14 years (95\% Cl: 9.5-18.5 years), ranging 0-61 years. Among these, $75.0 \%(n=42)$ were younger than 15 years. There were 21 boys and 21 girls, with a mean age of 5.7 years (95\% Cl: 4.5-6.8 years), ranging 0-15 years. Only 
25.0\% $(n=14)$ were older than 15 years. There were seven males and seven females, with a mean age of 38.9 years $(95 \%$ $\mathrm{Cl}$ : 29.8-48.0 years), ranging 17-61 years.

Monthly distribution showed that the highest number of JE cases occurred during June, July, and August, and the peak was in July (Figure 1). Yearly distributions showed that the mean number of JE cases was 4.9 (range 0-19) patients (Figure 2). The highest number of cases (19) was detected in 2016. Ten or more cases occurred in 2000, 2001, and 2014; however, no cases were detected in 2006 and 2007. Yearly distributions showed that the mean incidence rate (IR) for JE cases is $0.161 / 100,000$ population (95\% Cl: $0.09-0.24)$ (Figure 3). The highest incidence, $0.55 / 100,000$ population, was detected in 2016. The yearly distribution of incidence and cases corresponded with each other. Cumulative mapping (Figure 4) showed that JE was in high prevalence in the districts of the west and east coast divisions. The highest number of JE cases were reported in Kota Kinabalu $(n=23)$, with an IR of $0.72 / 100,000$ population, followed by Penampang $(n=13$, IR 1.94), Kota Marudu, and Tuaran ( $n=9$, IR 2.70 and 1.72), Keningau ( $n=8$, IR 1.02), Tenom ( $n=5$, IR 2.45), Tawau and Semporna ( $n=4$, IR 0.31 and 0.91), and Papar ( $n=3$, IR 0.85). In Kota Belud, Kudat, Nabawan, and Sandakan, there were two cases (IR 1.15, 1.20, 3.33, and 0.24) reported, and in Kuala Penyu, Ranau, Tambunan, Beluran, Pitas, and Kinabatangan, there was one case (IR 5.50, 1.40, 2.81, 1.32, 2.17, and 0.60) reported.

In 15 cases, pigs were present within a 400-m radius. In 36 cases, no pigs were present within a $400-m$ radius. In seven cases, the presence of pigs within a $400-\mathrm{m}$ radius was unknown. When the number of pig farms in each district was plotted against the cumulative map of JE cases, it was found that Tawau had the highest number of pigs, followed by Kota Kinabalu, Tuaran, Papar, Sandakan, and Penampang. Although Tawau had the highest number of pig farms, only four cases were reported in this district. We could not establish any relationship between the mapping of JE cases and the number of piggeries in each district. We could not establish a relationship between the average rainfall and the number of JE cases as the correlation coefficient was $0.27\left(r^{2}=0.0708\right)$.
The mortality varied from 0 to 4; from 2000 to 2005, one patient died of JE each year, but in 2014 alone, four patients died. Of 92 cases, a total of 10 (10.9\%) people died of JE.

\section{DISCUSSION}

The first cases of JE were confirmed by the Sabah State Health Department in 2000. In subsequent years, several cases were reported. The mean incidence of JE in Sabah is $0.161 / 100,000$ population, which is lower than the global overall incidence of $1.8 / 100,000$ population. ${ }^{1}$ However, we speculate that there might be more cases of JE in Sabah which go unnoticed because of limited awareness due to lack of information regarding the epidemiology of JE. Because rice cultivation and pig farming are not significant industries in Sabah, sporadic cases may occur without attracting the attention of healthcare professionals, who dismiss it as a significant infectious disease. The present study highlights the fact that people can become infected with JE where pigs are absent, despite the fact that the number of piggeries may not necessarily reflect the pig population. Nevertheless, unless a comprehensive study is carried out, it is difficult to indicate which animal was the amplifier in those cases or whether the pig population in Sabah is sufficient for maintaining JEV transmission. For instance, Culex tritaeniorhynchus, the major vector of JEV, can be carried long distances by winds. ${ }^{7}$ The Cx. tritaeniorhynchus-pig transmission cycle of JEV was first described in Japan at a time when pigs were intensively farmed and were the most numerous of possible, competent, vertebrate hosts. ${ }^{8}$ Yet not all regions of Asia experiencing JE outbreaks reflect this scenario. It is important to reconsider the transmission cycle of JEV, taking into account transmission contexts different from those first described in Japan. ${ }^{8} \mathrm{~A}$ series of studies showed that pigs play an important role as amplifying hosts for JEV in the neighboring state, Sarawak. ${ }^{9}$ However, in Singapore, the reservoir of JEV is likely to be aquatic birds because pig farming has been phased out in the early 1980 s. $^{10}$ Sabah has a rich population of aquatic birds; therefore, further study is needed to determine their role in JEV transmission.

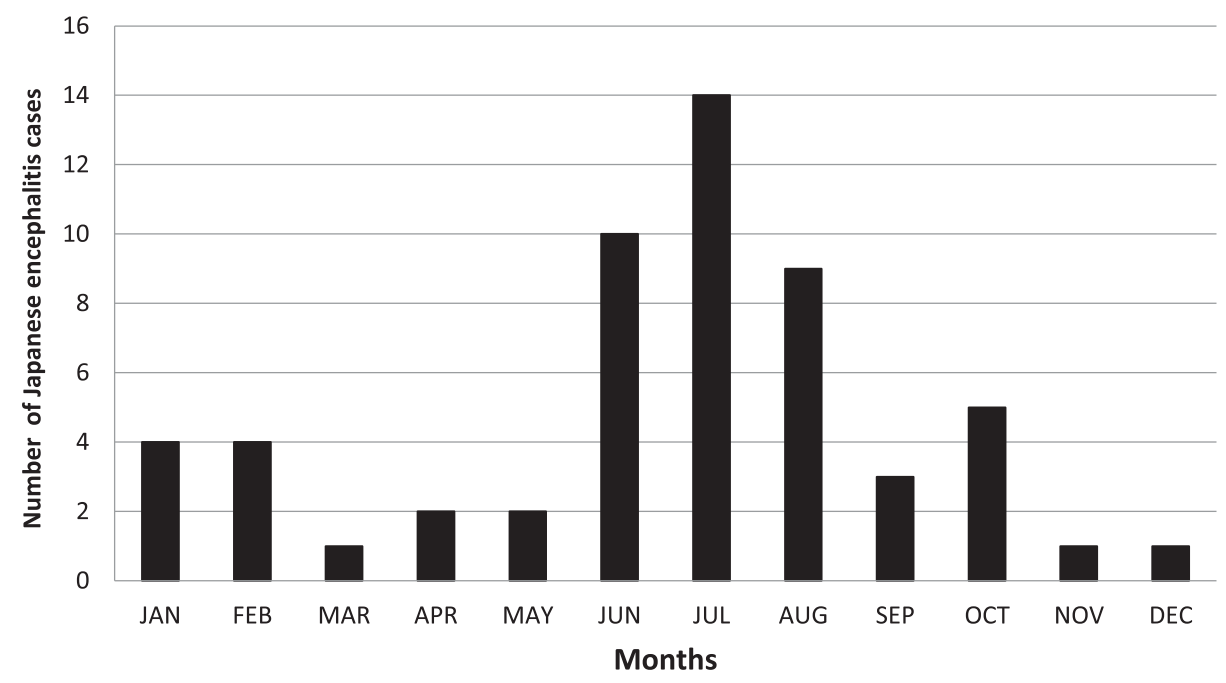

FIGURE 1. The seasonal occurrence of Japanese encephalitis (JE) cases in Sabah is represented by plotting the total number of JE cases for each month during the 19-year period. JE was predominant during June, July, and August, peaking in July. 


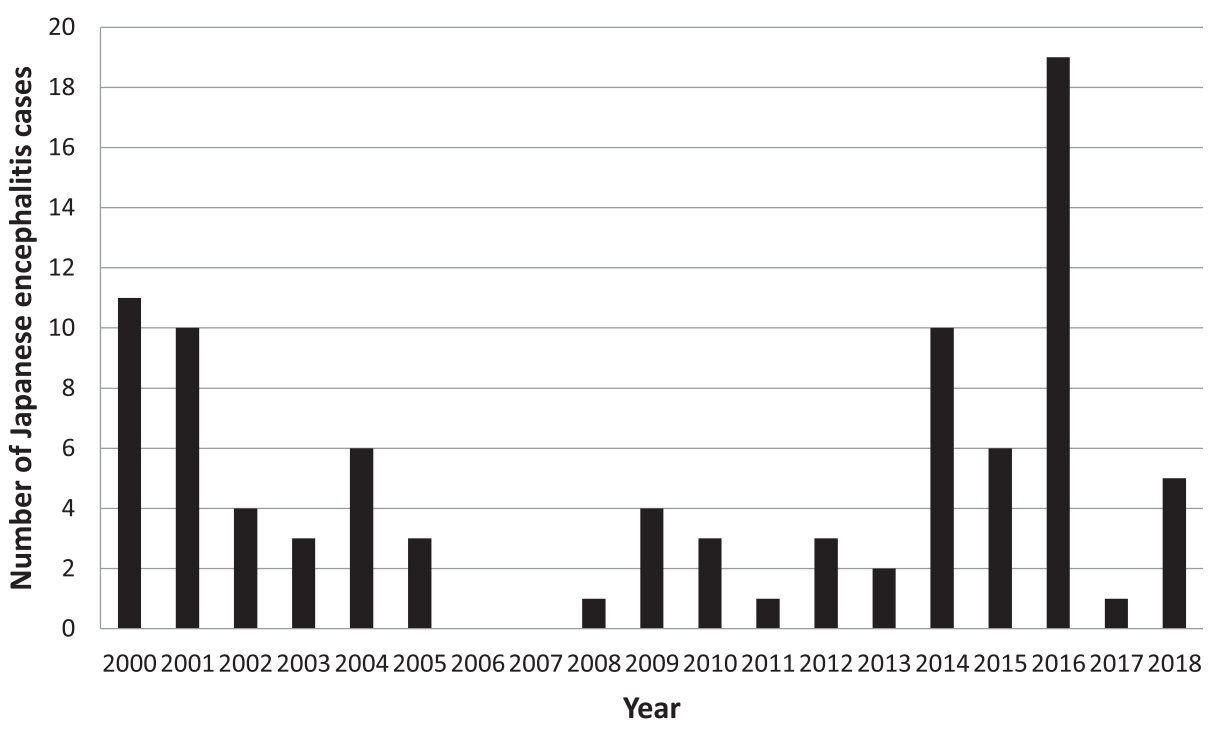

FigurE 2. Yearly distribution of Japanese encephalitis cases in Sabah from 2000 to 2018.

Similar to other endemic countries, most of JE cases in Sabah occurred in children younger than 15 years. ${ }^{11}$ However, the mortality rate we found in Sabah is considerably lower than the usual $20-30 \%$ mortality rate. ${ }^{12}$ In a study performed in the neighboring Sarawak state, the mortality was roughly half of that found in Sabah. ${ }^{12}$ Comparatively improved levels of facilities, such as diagnostic laboratory, life support system, and specialist doctors, were available for the JE patients in the tertiary care hospital where the study was performed in Sarawak, whereas our patients in Sabah were scattered in hospitals with different levels of facilities. We think that this might influence the mortality rate among patients with JE in Sabah. There was a yearly fluctuation in the number of JE cases in each district of Sabah. Although JE cases were prevalent throughout the year, the highest number of JE cases was identified in June, July, and August, peaking in July. We could not detect any correlation between rainfall in each district and the number of JE cases. In neighboring Sarawak, JE is also prevalent throughout the year, but most cases occurred from November to January, ${ }^{9}$ when the amount of rainfall is the highest. Sarawak has a similar climate to Sabah, only that the drier or wetter months start 1 or 2 months later. In Sabah, rain can be expected year-round, although, traditionally, the period between October and March is considered rainy and the period from April to September is considered as the dry season. However, rain can vary depending on the mountain and coastal areas and also the effects of other variables, such as the occurrence of typhoons and El Nino. Therefore, a more comprehensive study is needed to determine the effects of rain on the occurrence of JE in Sabah.

A limitation of this study is that neuropsychological adverse effects on the survivors were not documented. In Sarawak, more than half of the patients continued to experience neuropsychological sequelae and behavioral disorders. ${ }^{12}$ Long-term neurological follow-up of JE patients is needed in Sabah to fully elucidate these complications and to provide more care to people. It might be recommended to introduce a JEV vaccine in Sabah, although the determination of the circulating genotypes is necessary because the current JE vaccine has low protective efficacy against the emerging genotype 5 JEV. ${ }^{13}$ Therefore,

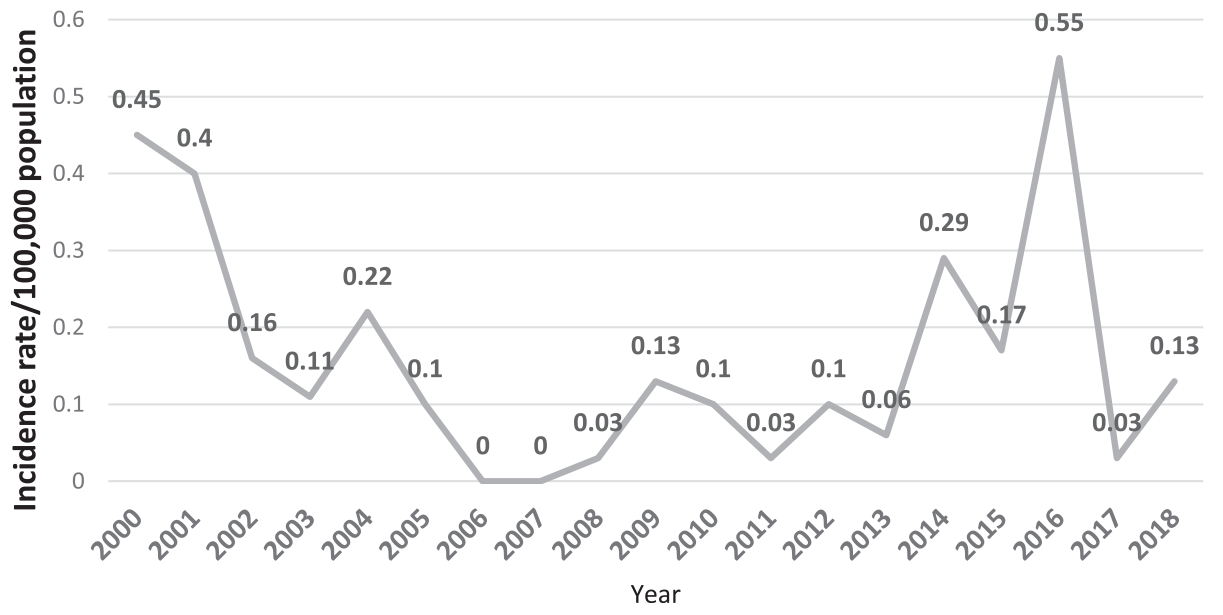

FIGURE 3. Yearly distribution of incidence rate of Japanese encephalitis cases in Sabah from 2000 to 2018. The mean incidence rate is $0.161 /$ 100,000 population (95\% Cl: 0.09-0.24). 


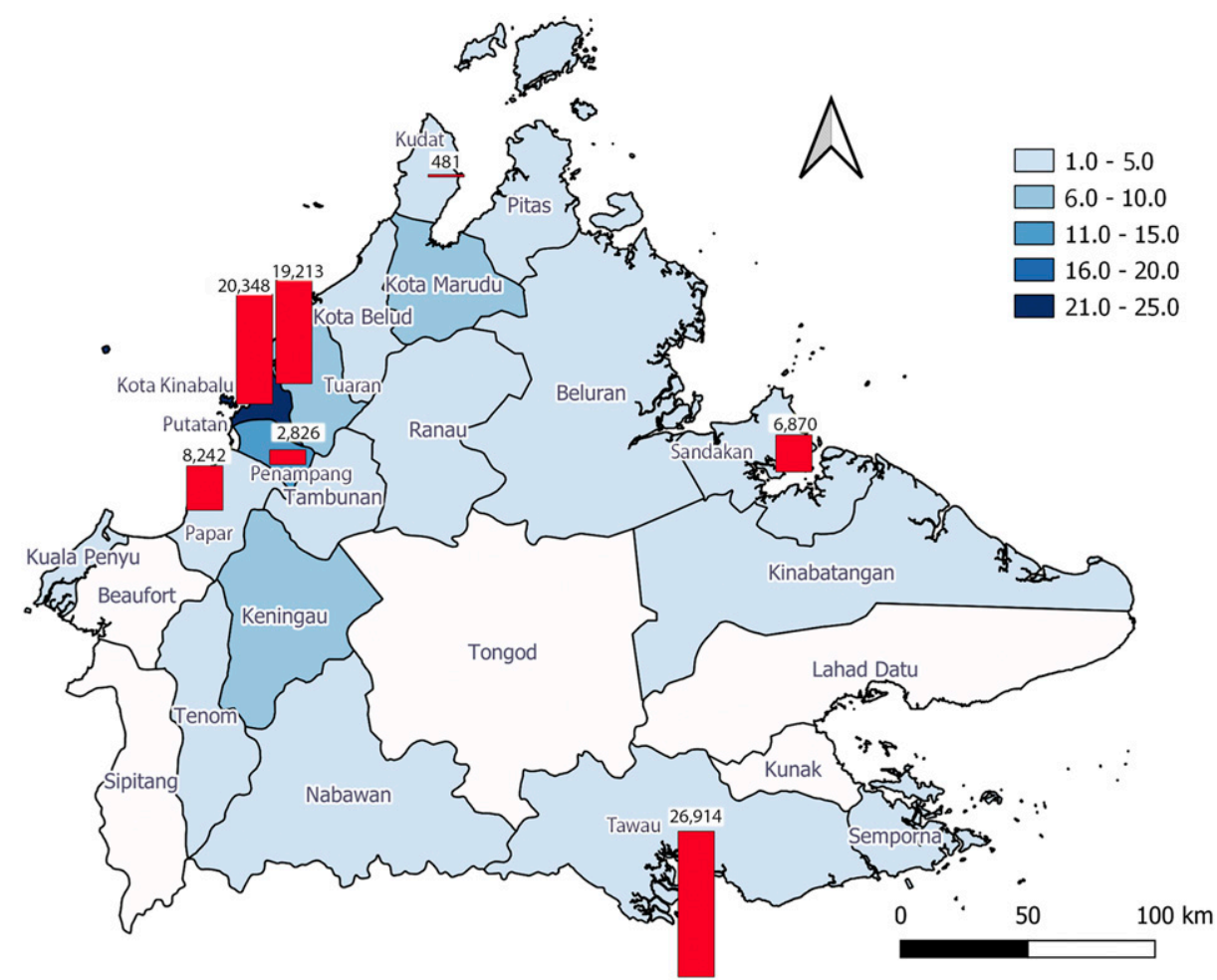

FIGURE 4. The cumulative number of Japanese encephalitis cases and the number of pig farms in each district of Sabah are plotted on the map. The number of pig farms is indicated by the red column (count on top of the column).

before introducing the JEV vaccine in Sabah, a full-scale study on the seroprevalence and genotype distribution of JEV is needed.

\section{CONCLUSION}

This retrospective study from the notification of the last 19 years revealed that JE is a challenge in Sabah and children are the most vulnerable group affected by this infection, as expected. The number of cases varied yearly and mainly occurred in June, July, and August, peaking in July (which corresponds to the dry season in Sabah). We could not find a relationship between the number of JE cases and the amount of rainfall or the number of pig farms. In a considerable number of cases, no pig farm was in a 400-m radius of the residence. These data indicate that more studies are needed to better understand the full picture of JE in Sabah.

Received December 14,2019. Accepted for publication April 20, 2020.

Published online June 8, 2020.

Acknowledgments: We thank the director general of the Ministry of Health, Malaysia for the permission to publish this article. We also thank all the healthcare personnel of Sabah State Health Department who were involved in the diagnosis, treatment, management, and reporting the Japanese encephalitis cases.

Financial support: The study was funded by launching grant for Centre of Excellence from Universiti Malaysia Sabah (number: AM 18006).

Authors' addresses: Marilyn Charlene Montini Maluda, Mohd Yusof Ibrahim, and Mohammad Saffree Jeffree Department of Community and Family Medicine, Faculty of Medicine and Health Sciences, Kota Kinabalu, Malaysia, E-mails: marilynmaluda@gmail.com, dr.myusof@ ums.edu.mu, and saffree@ums.edu.my. Jenarun Jelip, Ministry of Health Malaysia, Vector Borne Disease Sector, Putrajaya, Malaysia, E-mail: jenarun@moh.gov.my. Maria Suleiman, Ministry of Health
Malaysia, Disaster, Outbreak, Crisis and Emergency Sector, Putrajaya, Malaysia, E-mail: mariasuleiman@moh.gov.my. Azalina Farina Binti Aziz and Jaeyres Jani, Borneo Medical and Health Research Centre, Faculty of Medicine and Health Sciences, Universiti Malaysia Sabah, Kota kinabalu, Malaysia, E-mails: azalinafarina@yahoo.com and jaeyres96322@gmail.com. Takaaki Yahiro, Department of Pathobiology and Medical Diagnostics, Faculty of Medicine and Health Sciences, Universiti Malaysia Sabah, Kota kinabalu, Malaysia, E-mail: takaaki_826 111@yahoo.co.jp. Kamruddin Ahmed, Department of Pathobiology and Medical Diagnostics, Faculty of Medicine and Health Sciences, Universiti Malaysia Sabah, Kota Kinabalu, and Malaysia and Borneo Medical and Health Research Centre, Faculty of Medicine and Health Sciences, Universiti Malaysia Sabah, Kota Kinabalu, Malaysia, E-mail: ahmed@ ums.edu.my.

\section{REFERENCES}

1. Campbell GL et al., 2011. Estimated global incidence of Japanese encephalitis: a systematic review. Bull World Heal Organ 89: 766-744.

2. Morita K, Nabeshima T, Buerano CC, 2015. Japanese encephalitis. Rev Sci Tech 34: 411-452.

3. Oda K, Igarashi A, Kheong CT, Hong CC, Vijayamalar B, Sinniah M, Hassan SS, Tanaka H, 1993. Cross-sectional serosurvey for Japanese encephalitis specific antibody from animal sera in Malaysia. Southeast Asian J Trop Med Public Health 27: 463-470.

4. Wolfe ND, Kilbourn AM, Karesh WB, Rahman HA, Bosi EJ, Cropp BC, Andau M, Spielman A, Gubler DJ, 2001. Sylvatic transmission of arboviruses among Bornean orangutans. Am J Trop Med Hyg 64: 310-316.

5. World Health Organization, 2018. Vaccine Preventable Diseases Surveillance Standards, Japanese Encephalitis. Available at: https://www.who.int/immunization/monitoring_surveillance/ burden/vpd/WHO_SurveillanceVaccinePreventable_10_JE_ R2.pdf?ua=1. Accessed March 21, 2020.

6. Yang DK, Kweon CH, Kim BH, Lim SI, Kim SH, Kwon JH, Han HR, 2004. TaqMan reverse transcription polymerase chain reaction for the detection of Japanese encephalitis virus. $J$ Vet Sci 5: 345-351. 
7. Nabeshima T, Loan HT, Inoue S, Sumiyoshi M, Haruta Y, Nga PT, Huoung VT, del Carmen Parquet M, Hasebe F, Morita K, 2009. Evidence of frequent introductions of Japanese encephalitis virus from south-east Asia and continental east Asia to Japan. $J$ Gen Virol 90: 827-832.

8. Lord JS, Gurley ES, Pulliam JR, 2015. Rethinking Japanese encephalitis virus transmission: a framework for implicating host and vector species. PLoS Negl Trop Dis 9: e0004074.

9. Wong SC et al., 2008. A decade of Japanese encephalitis surveillance in Sarawak, Malaysia: 1997-2006. Trop Med Int Health 13: 52-55.

10. Ting SH, Tan HC, Wong WK, Ng ML, Chan SH, Ooi EE, 2004. Seroepidemiology of neutralizing antibodies to Japanese encephalitis virus in Singapore: continued transmission despite abolishment of pig farming? Acta Trop 92: 187-191.

11. Lopez AL, Aldaba JG, Roque VG Jr., Tandoc AO 3rd, Sy AK, Espino FE, DeQuiroz-Castro M, Jee Y, Ducusin MJ, Fox KK, 2015. Epidemiology of Japanese encephalitis in the Philippines: a systematic review. PLoS Negl Trop Dis 9: e0003630.

12. Ooi MH et al., 2008. The epidemiology, clinical features, and longterm prognosis of Japanese encephalitis in central Sarawak, Malaysia, 1997-2005. Clin Infect Dis 47: 458-468.

13. Cao Let al., 2016. Low protective efficacy of the current Japanese encephalitis vaccine against the emerging genotype 5 Japanese encephalitis virus. PLoS Negl Trop Dis 10: e0004686. 